\title{
A Human-in-the-Loop Evaluation of Air-Ground Trajectory Negotiation
}

\author{
Nancy M. Smith* and Paul U. Lee \\ NASA Ames Research Center and San Jose State University Foundation, Moffett Field, CA, 94035-1000 \\ Thomas Prevôt ${ }^{\ddagger}$ and Joey Mercer ${ }^{\S}$, \\ San Jose State University Foundation at NASA Ames Research Center, Moffett Field, CA, 94035-1000 \\ Everett A. Palmer, III**, V. Battiste ${ }^{\dagger \dagger}$ and W. Johnson ${ }^{*}$ \\ NASA Ames Research Center, Moffett Field, CA, 94035-1000
}

\begin{abstract}
An integrated air ground simulation with commercial airline pilots and certified professional controllers was conducted at NASA Ames Research Center to evaluate a concept for air-ground trajectory negotiation. This concept was developed as part of the Distributed Air-Ground Trajectory Negotiation Project, which explores use of new technology, including CPDLC and flight deck and ATC decision support tools, to accommodate user preferred trajectories. Two human-in-the-loop simulation studies were conducted in 2002 and 2003. The first study in 2002 focused on how an integration of air and ground side decision support tools (DSTs) with data link can potentially improve efficiency, capacity, and workload distribution. The second study in 2003 focused on pilot/controller interactions during a trajectory negotiation. The results from the 2002 study suggested that this concept allowed for more precise delivery, efficient flight paths, and lower controller workload, while the 2003 study demonstrated the feasibility of trajectory negotiation via data link. This paper summarizes these results, discusses critical factors that contribute to the success of the concept, and open issues that need to be understood in order to further the concept. Overall, integration of DSTs and data link seems to show great potential. The trajectory negotiation concept appears feasible but its potential for benefits needs further research.
\end{abstract}

\section{Introduction}

\section{A. Background}

This paper describes results from a pair of human-in-the-loop (HITL) simulations conducted at the National Aeronautics and Space Administration's (NASA's) Ames Research Center to investigate a concept for improving efficiency in the National Airspace System (NAS) through air-ground trajectory negotiation. This concept was developed as part of Distributed Air-Ground Traffic Management (DAG-TM), a project that explores use of new automation and communication technologies to modify the roles and responsibilities of NAS users and service providers. ${ }^{1,2}$ The goal of DAG-TM is to facilitate user-preferred routing, increase system flexibility and capacity, and improve NAS operational efficiency by developing a concept that supports collaboration at all levels of traffic management decision-making. The DAG-TM concept leverages off technological and procedural innovations including communication, navigation, and surveillance / air traffic management (ATM) technologies; air and ground decision support tools (DSTs); and distributed separation responsibility. This paper describes work conducted to

\footnotetext{
* Research Psychologist, Human-Automation Integration Research Branch, MS262-4. AIAA member.

$\dagger$ Senior Research Associate, Human-Automation Integration Research Branch, MS262-4.

$¥$ Senior Research Engineer, Human-Automation Integration Research Branch, MS262-4. AIAA member.

${ }^{\S}$ Research Associate, Human-Automation Integration Research Branch, MS262-4.

${ }^{* *}$ Human Factors Engineer, Human-Automation Integration Research Branch, MS262-4.

${ }^{\dagger \dagger}$ Research Psychologist, Human Information Processing Research Branch, MS262-2. AIAA member.

${ }^{\ddagger}$ Research Psychologist, Human Information Processing Research Branch, MS262-2.
} 
develop and evaluate a DAG-TM concept element (CE 6) for en route trajectory negotiation. ${ }^{3}$ It was completed as part of the Advanced Air Transportation Technologies (AATT) project under NASA's Airspace Systems program.

The objectives of CE 6 are to reduce unnecessary and/or excessive air traffic service provider (ATSP) issued route deviations, reduce controller workload, and facilitate trajectory change requests. CE 6 proposes several means to achieve these objectives that can be broadly characterized as integration of controller and user automation with data link. The capability to negotiate trajectory changes between users and controllers during flight is an added benefit supported by this integration. These negotiations may be initiated by the controller, flight deck, or the airline operations center (AOC).

\section{B. Integration of data link with air and ground automation}

CE 6 explores the potential for improving local airspace efficiency through data link-automation integration on a number of different levels. The trajectory prediction accuracy of controller DSTs can be greatly enhanced through user-supplied data on key flight parameters, which may be broadcast or sent as an addressed message from flight deck to ground automation. Trajectory prediction improvements directly affect the controller's decisions related to separation assurance and metering, and should help reduce unnecessary and/or excessive controller issued route deviations and improve the overall effectiveness of controller operations.

Integration of controller automation with an ability to send addressed messages to the flight deck also provides a number of advantages. These include:

- fewer voice communications, reducing frequency congestion in high density airspace

- uplink messages cam be automatically generated or prepared by controller decision support tools (DSTs), reducing controller workload

- complex or multi-part uplink message content can be stored, printed or reviewed by the flight crew.

Integrating flight deck automation with data link can provide the ability to load uplinked addressed message content into the appropriate on-board systems (e.g., tune radios to assigned frequency, input complex clearances into flight management system (FMS), input revised wind forecasts into the FMS). This integration has significant advantages, including:

- improving flight deck trajectory prediction accuracy and consistency with ground predictions

- enabling the transmission and execution of complex trajectory changes during flight

- reducing the opportunities for human error inherent in manual data entry

- reducing flight crew workload.

Providing the flight deck and the AOC the ability to submit user-preferred trajectories through data link facilitates user-initiated trajectory change requests, and should reduce controller workload associated with accommodating user preferences. If the user has access to current TFM constraints and the tools to construct conflict-free trajectories the potential for request acceptability is increased.

Prototype implementations of CE 6 flight deck and controller DSTs and procedures were iteratively developed and tested in NASA Ames' Flight Deck Display Research Laboratory (FDDRL) and Airspace Operations Laboratory (AOL) ${ }^{4-7}$ This document provides a human factors assessment of the CE 6 concept based on results and observations from two human-in-the-loop simulations, conducted in the FDDRL, AOL and Ames Advanced Concepts Flight Simulator, in September $2002^{8,9}$ and November 2003. ${ }^{10}$

\section{Implementation}

\section{A. Data link}

Data link capabilities in this simulation environment include simulated controller-pilot data link communication (CPDLC) and Automatic Dependent Surveillance-Broadcast (ADS-B) flight information broadcasting. CPDLC supports exchange of point-to-point addressed messages between the flight deck and the air traffic controller. The CPDLC message set includes uplink messages (controller-to-pilot) for radio frequency assignment, RTA assignment, altitude clearance, speed clearance, trajectory clearance, descent forecast winds, and responses to downlink message requests. Downlink messages (pilot-to-controller) include trajectory requests and responses to uplink messages. Message sending may be automated (uplink TOC message when handoff is accepted, uplink forecast winds on sector entry) or user-initiated (downlink route requests, uplink clearances).

The ADS-B simulation includes $1 \mathrm{~Hz}$ broadcast of aircraft state data (latitude, longitude, altitude, heading, speed) and transmission of the FMS trajectory whenever it changes (a trajectory change point was sequenced, or the flight crew modified the FMS route). 


\section{B. Ground Automation}

The controller DSTs and interface for CE 6 simulations evolved during the course of the project. The platform used in 2002 was a version of the Center TRACON Automation System (CTAS) plan-view graphical user interface (PGUI) modified by DAG-TM researchers to support CE 6 concept exploration. ${ }^{7,11}$ It included a color-coded radar display with a conflict list, route trial planning, time-based metering with an interactive timeline and speed advisories, and a custom CPDLC interface (Figure 1).

CPDLC-ground automation integration enabled:

- route trial plans, altitude trial plans and meet-time speed advisories to be sent to aircraft as automatically formatted route, altitude or speed uplink clearances

- metering RTAs (required time of arrival clearances) to be uplinked

Aircraft information provided through data link was used by ground automation in both simulations as follows:

- current (1 second update) ADS-B altitude and speed were presented in the data block

- current ADS-B heading was used for trend vector lines

- current ADS-B lat-long coordinates determined aircraft target location (though "history" markers were refreshed at the conventional 12 second radar update rate)

- current ADS-B state data (altitude, heading, speed, latitude, longitude) was combined with current FMS intent information to improve the trajectory predictions used in conflict detection and in determining ETAs for metering.

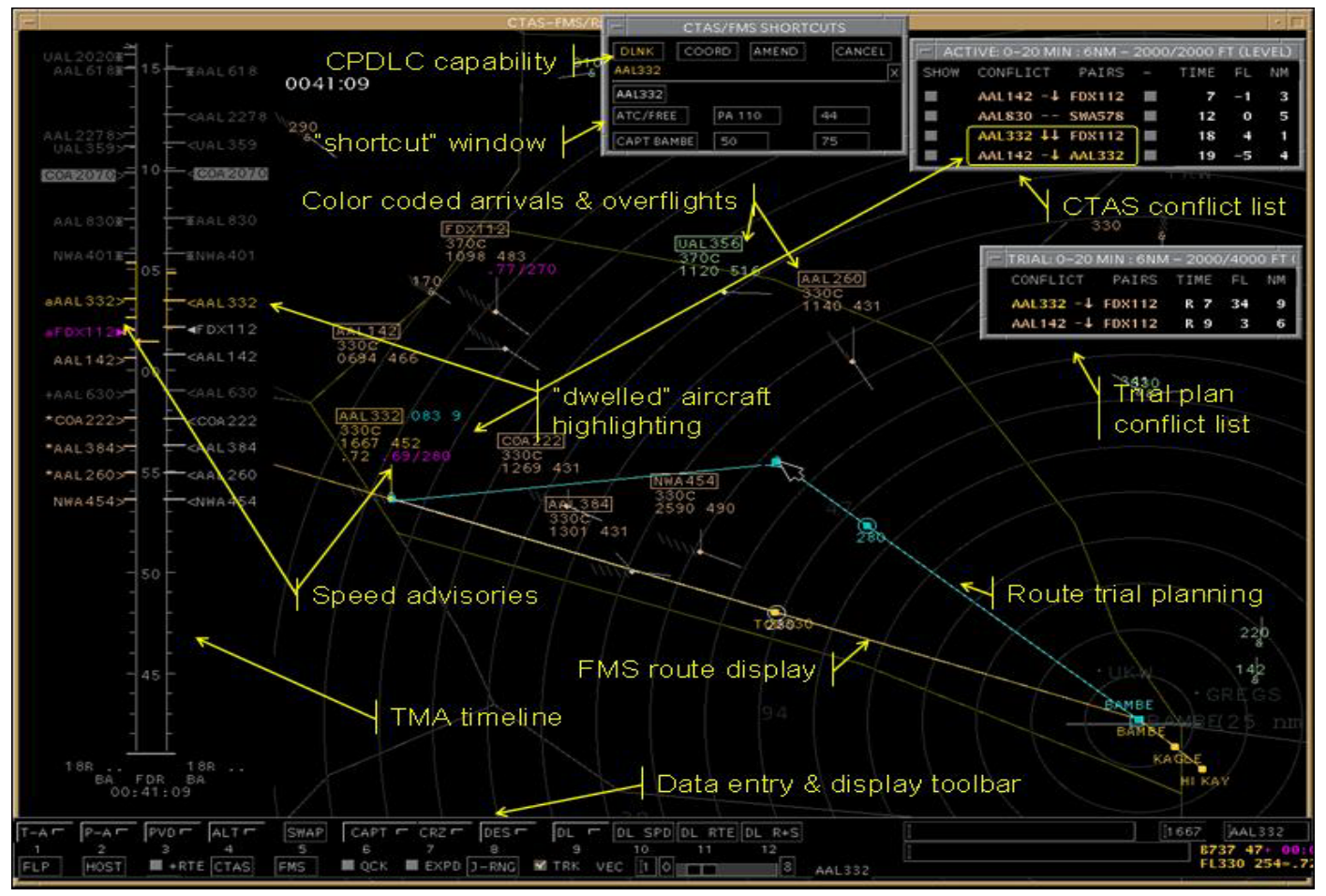

Figure 1. 2002 En Route Controller Display, CTAS implementation.

In the second simulation, controller DSTs were integrated into a version of the Multi-Aircraft Control System (MACS) that can be configured to emulate the Display System Replacement (DSR) workstation used in today's en route ATC facilities. ${ }^{6,12}$ Conflict prediction and route trial planning in MACS/DSR were modified from the earlier design to incorporate data block display and control elements patterned after the CTAS Direct-to interface. ${ }^{13,14}$ The MACS/DSR CPDLC interface was modeled after CPDLC Build I (used in Miami Center). ${ }^{15}$ Its features include data block symbology, automated transfer-of-communication (TOC), a status list, and host computer input functions (Figure 2).

New CPDLC functions were developed in MACS/DSR for the 2003 simulation to support pilot-controller negotiation of flight deck trajectory change requests. Although this task is not supported in CPDLC Build I, interface enhancements for handling downlinked requests were designed to be consistent with the look and feel of the existing system (Figure 3). 


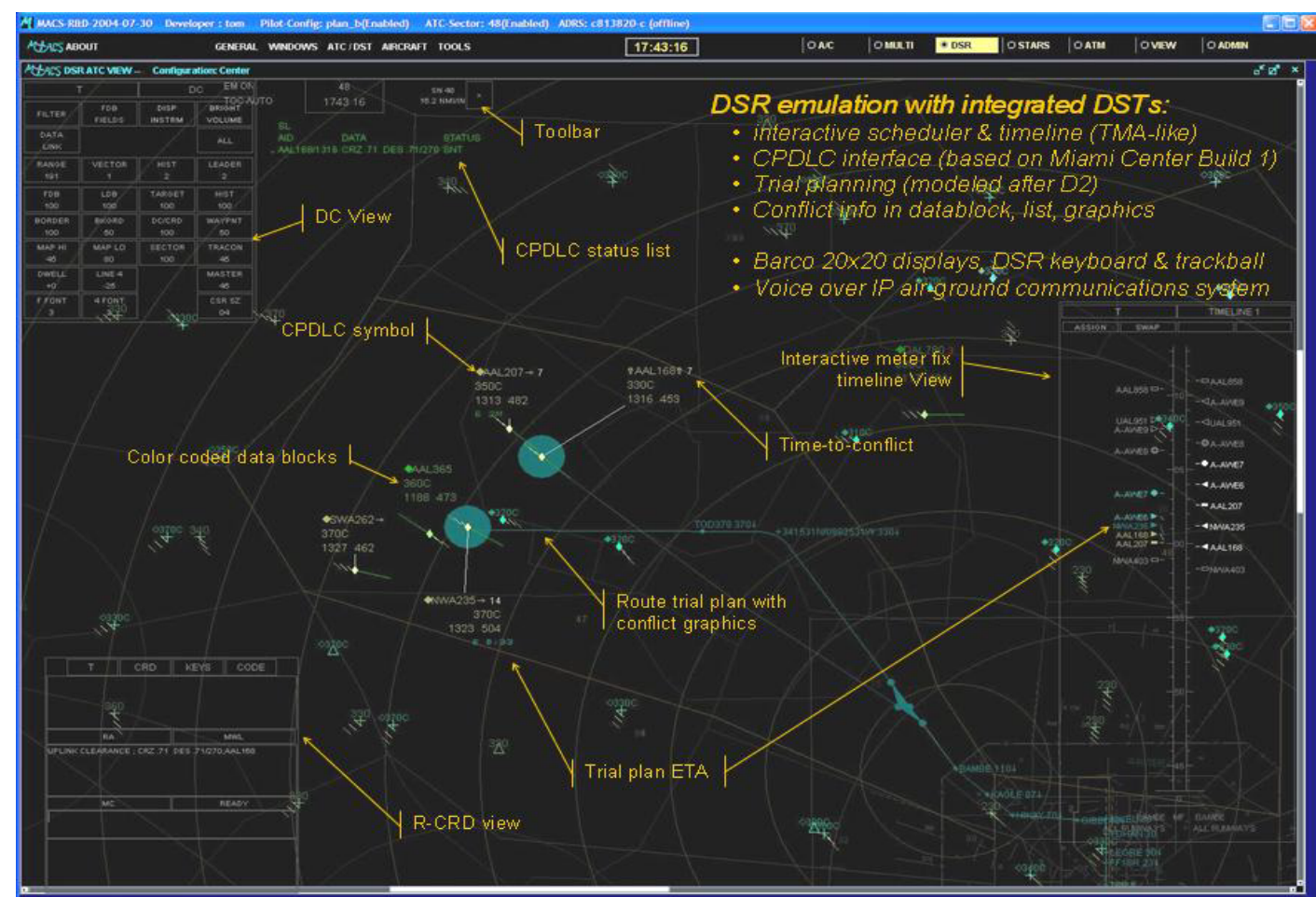

Figure 2. 2003 En Route Controller Display, MACS/DSR implementation.
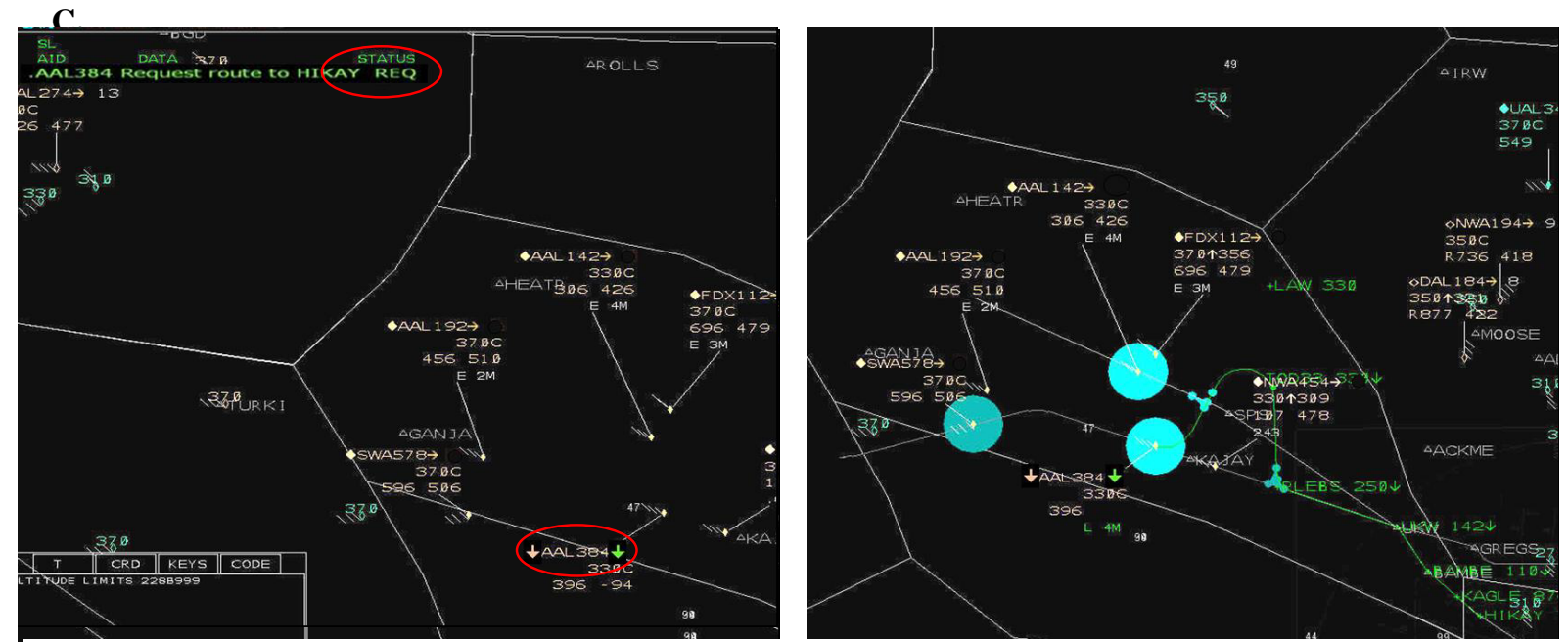

Figure 3. MACS/DSR downlink request display example (2003).

Ground automation has received a donwlink request from AAL384. Red circles indicate controller cues (status list entry and down arrows in data block). On the right, the requested route (accessed by clicking on the green arrow) and ground automation feedback on request-related conflicts and meter fix delay are shown. 


\section{Flight Deck Automation}

The flight crew's DST interface for both simulations was a cockpit display of traffic information, or 3D-CDTI (Figure 4) The 3D-CDTI included several specialized flight management tools for facilitating trajectory negotiation:

- Proximal traffic location, their current status, and flight plan data

- Strategic conflict detection and alerting

- Automated conflict resolution strategies

- The facility to graphically plan manual route changes

- Time-based, in-trail spacing on approach.

The traffic display provided pilots not only with aircraft state (e.g., track, ground speed, and altitude), but also with 4D intent information needed to help flight crews maintain a high level of traffic awareness and allowed them to evaluate automated resolutions. Flight path could be viewed and future position over time could be shown with pulses running along planned paths of travel. Pilots could also display traffic in a three-dimensional (3D) perspective view.
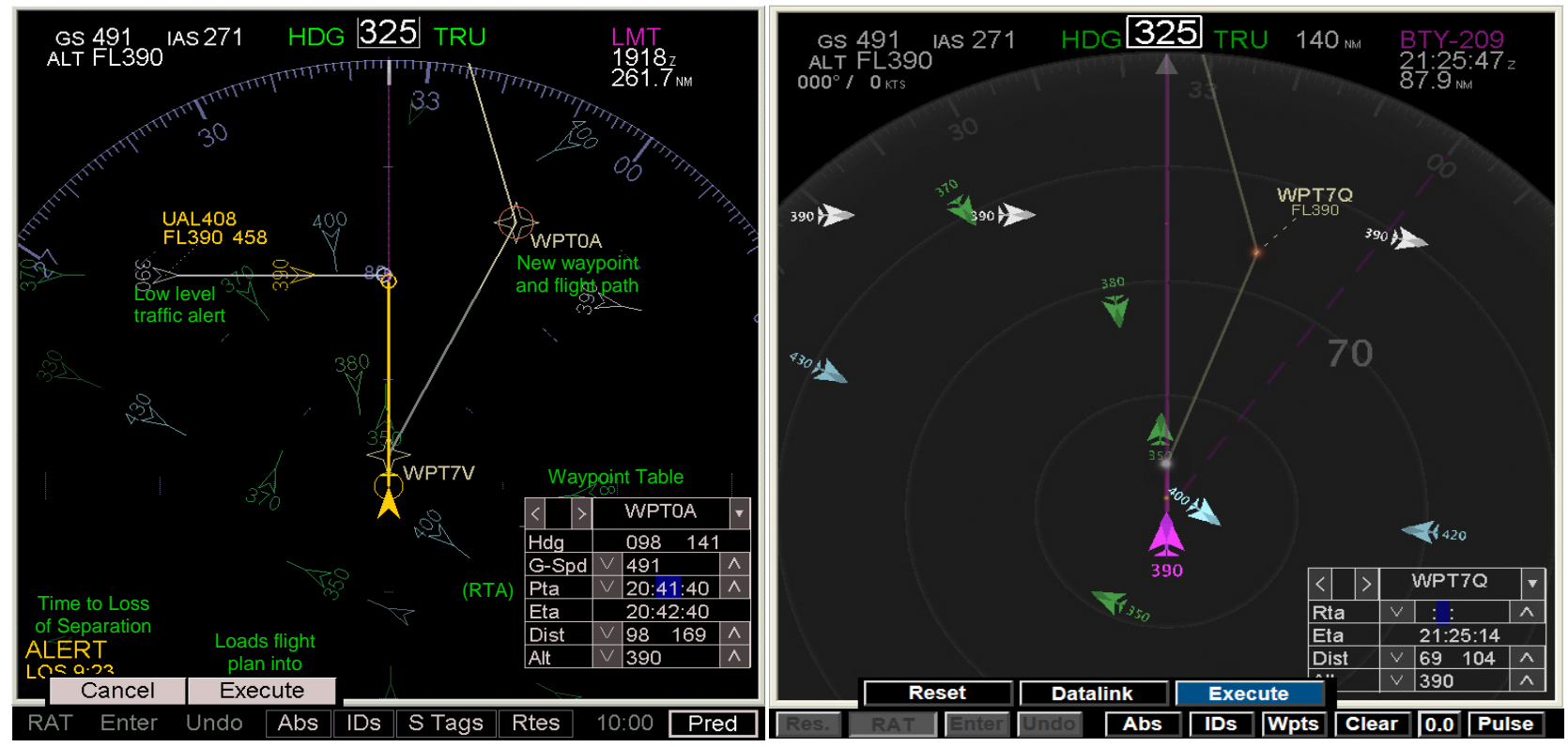

Figure 4. Flight Deck display examples: 2002 (left), 2003 (right).

\section{Simulation Results}

\section{A. September 2002}

In September 2002 NASA's AATT project office sponsored a human-in-the-loop simulation to examine three concept elements for integrated air and ground operations. ${ }^{16}$ One of the concept elements explores the viability and potential benefits of flight deck initiated trajectory changes by collecting system-level and human performance measures. Participants included five certified professional controllers and eight commercial pilots who operated in scenarios that simulated Dallas/Fort Worth International Airport (DFW) arrivals approaching from the northwest. As part of each scenario, aircraft flew through en route airspace, transitioned into the Terminal Radar Approach Control (TRACON), and landed at DFW. Participants were instructed to meet a required time of arrival (RTA) at a meter fix located at the TRACON boundary. Trials varied according to the level of advanced technologies and procedures, and included a baseline condition that represented current-day operations.

For the baseline, controllers had available an arrival scheduler, but without any added DSTs. Pilots had cockpit displays of traffic information (CDTI), but were not permitted to request trajectory changes. For the CE 6 condition, flight deck simulators were equipped with CDTI that had conflict detection and resolution (CD\&R) logic and RTA capability. Controller DSTs included the Center-TRACON Automation System (CTAS) Traffic Management Advisor (TMA), a timeline presenting the TMA meter fix schedule, a trajectory-based conflict probe, trial planning capability, color enhancements to the traffic display, and controller pilot datalink communication (CPDLC). All flights were under positive ground control, however, pilots were allowed to negotiate route changes with controllers 
via CPDLC. Pilot and controller performance was evaluated based on several human performance metrics including usability, suitability, acceptability, and workload. System-level metrics, including capacity, complexity, efficiency, and safety were also evaluated.

In general, CE 6 showed benefits mechanisms for efficiency and capacity without compromising safety or significantly increasing workload. ${ }^{6,8,9}$ Under CE 6, aircraft flew more efficient paths at higher altitudes over a shorter period of time. With a target spacing interval of 82 seconds per aircraft, the CE 6 condition provided a $5.5 \%$ increase over baseline meter fix throughput (from 41.6 to 43.9 aircraft per hour; Figure 5).

In addition, aircraft were delivered to the TRACON meter fix more accurately and with better spacing in CE 6 than in the baseline condition (Figure 6).

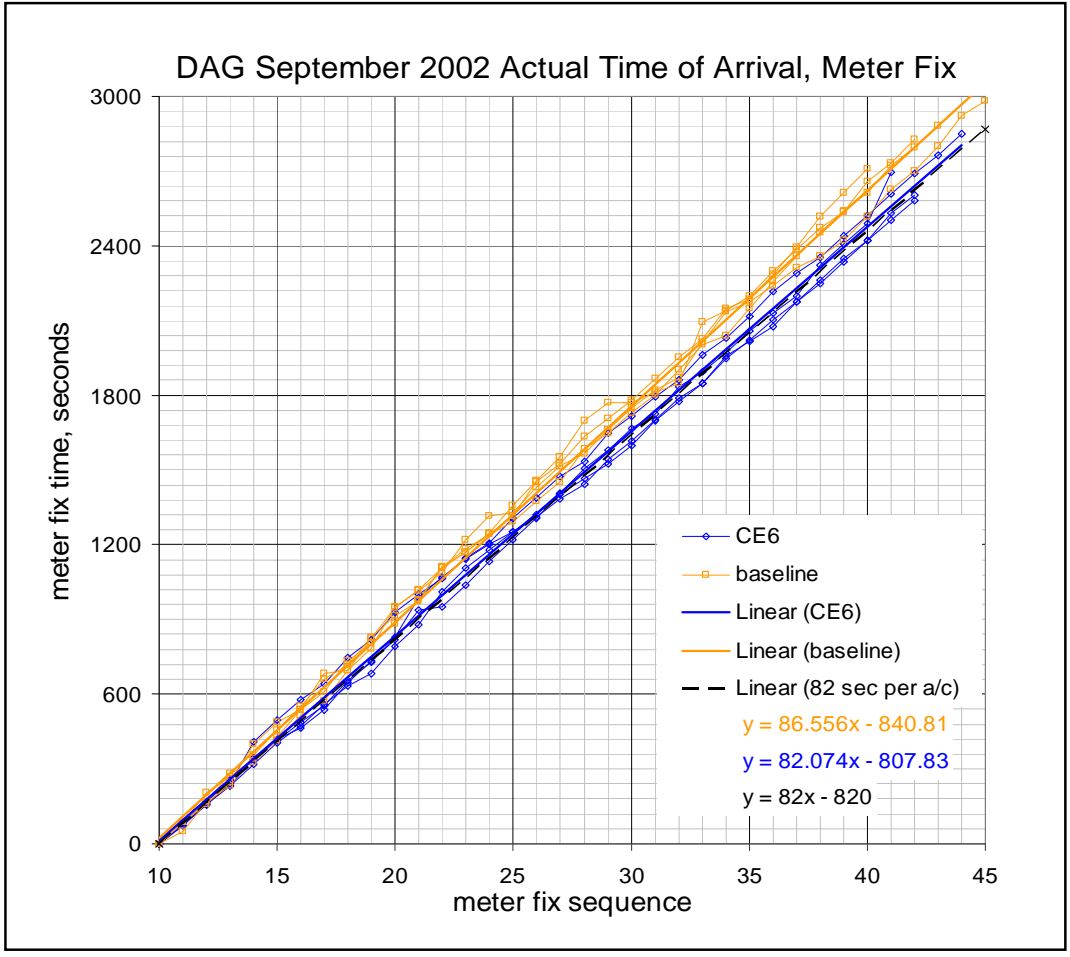

Figure 5. Meter fix throughput for Baseline and CE 6, 2002 simulation. The mean spacing interval observed during the simulation was 82.074 seconds in the CE 6 condition and 86.556 seconds in the Baseline condition. The target spacing interval was 82 seconds. Data for each run is normalized for crossing time of $10^{\text {th }}$ aircraft.
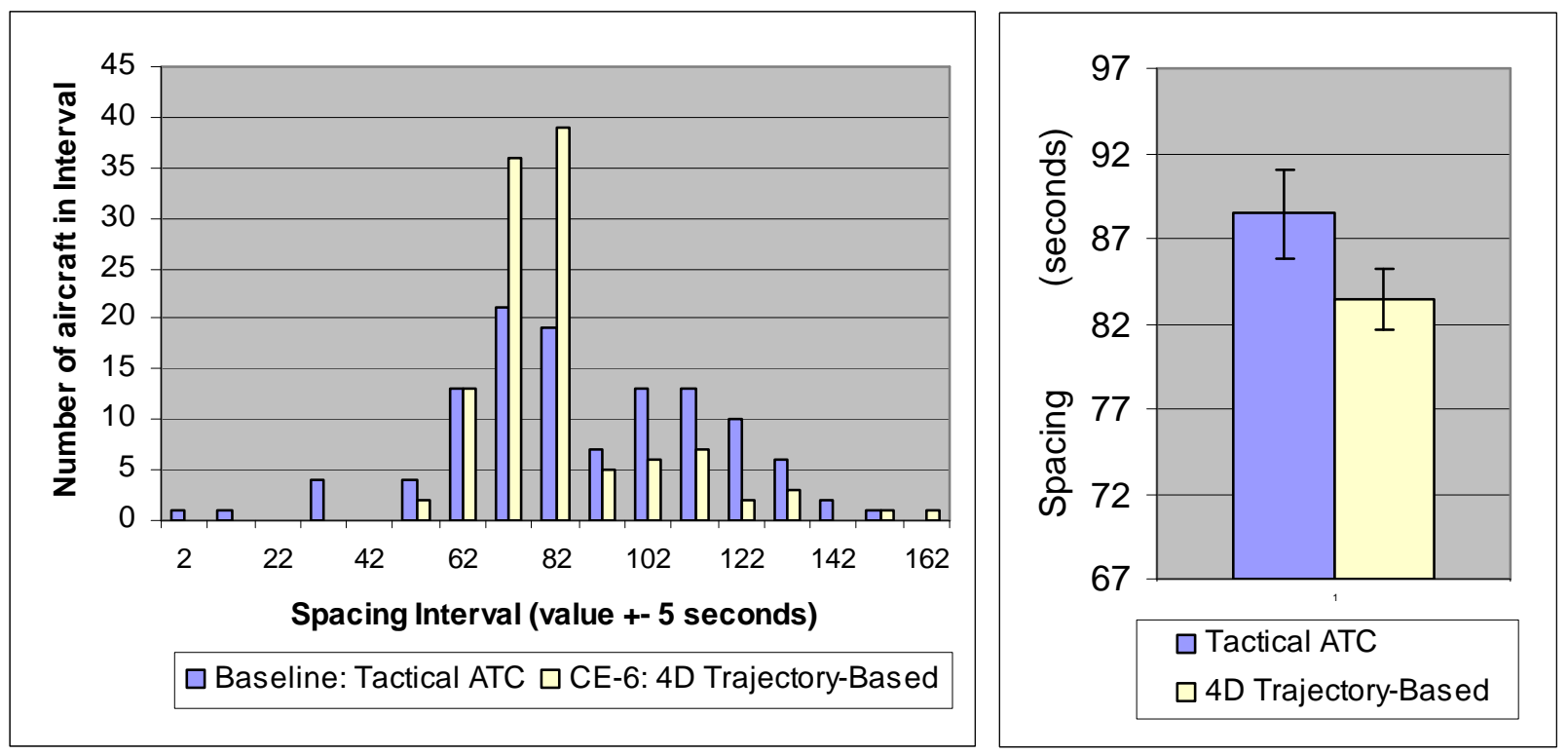

Figure 6. Inter-Arrival Spacing at the Meter Fix for Baseline and CE 6 operations, 2002 simulation.

Inter-Arrival Spacing Histogram (left), Inter-Arrival Spacing Mean and Standard Error (right) In the trajectorybased condition (CE-6) more aircraft were delivered within the given tolerance (+/- 15 seconds) of their target spacing (82 seconds) and the variance was significantly reduced. 
Subjective feedback from the participants further indicated the acceptability of the concepts in terms of mental workload, temporal demand, and situation awareness, as compared to operating with current day equipment (Figure7).

Results from this simulation clearly demonstrated potential benefits of integrating air and ground automation with data link and addressed safety concerns. The feasibility of negotiated trajectory requests was not explored in depth, however, and that became the focus of the 2003 simulation.

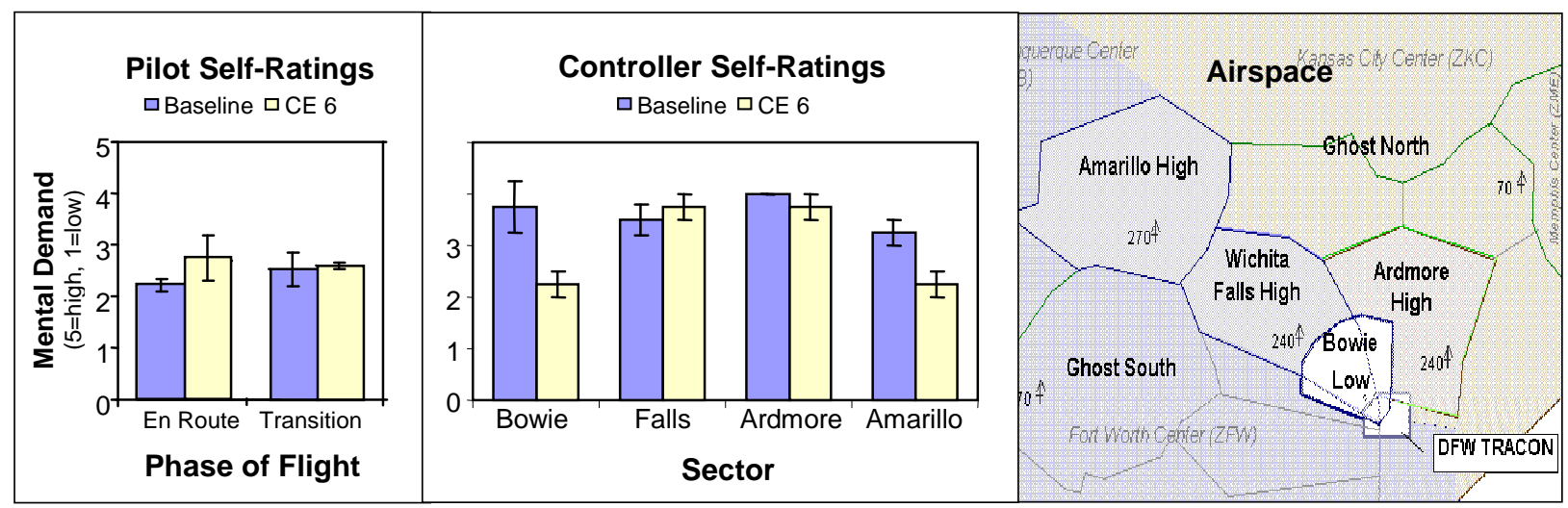

Figure 7. Pilot and controller workload (mental demand), 2002 simulation.

Pilot self-ratings of mental demand increased in CE 6, but never exceeded a "moderate" value (left graph). Controller ratings (middle graph) indicated that workload was re-distributed from downstream to upstream sectors under CE 6, with the BOWIE low altitude arrival controller reporting the greatest benefit.

\section{B. November 2003 Simulation}

In November 2003 a second HITL simulation was conducted to evaluate procedures and tools for air-ground negotiation of flight deck trajectory requests. ${ }^{10}$ In this study, improved controller MACS/DSR display and 3-D cockpit displays were used (Figures 2, 4). The controller display incorporated improved DSTs including a better route trial planning tool, speed advisories, and the TOC operations found in CPDLC I. Flight deck tools were also updated with better conflict detection capabilities and optional 3-D views that can be rotated to view from any angle.

Trajectory changes requested by the flight deck were made either by voice or by CPDLC, depending on the experimental condition. The controller could respond to the request by voice or CPDLC, as appropriate. A pilot request sent by CPDLC was presented to the controller in two ways: a down arrow symbol in the data block that is clickable to view the requested route and a "REQ" message in the data link status list (see Figure 3). The request can be accepted or rejected either by voice or by CPDLC, but if the request was rejected, a radio communication explaining the reason for rejecting the request was required, providing the opportunity for the pilot to reformulate a more acceptable request, or for the controller to determine flight crew intent and offer an alternative solution.

For each run, pilots or controllers were given scripted reasons for changing the route of every subject-piloted aircraft (e.g., a direct routing request, or unusually large delay) that could result in a "user-preferred" solution developed on the flight deck. Each of these "route request probes" was repeated in four different conditions to determine the effect of supporting air and ground technology. The four conditions were:

1) Baseline (CPDLC for TOC, metering with timeline, no other advanced tools)

2) CE-6 Uplink Only (added ground conflict probe, uplink speed advisory and route clearances)

3) CE-6 Uplink \& Downlink (added flight deck Route Assessment Tool without CD\&R)

4) CE-6 Uplink \& Downlink (added flight deck CD\&R)

Participants included four certified professional center controllers and ten commercial airline pilots. Controllers worked three high altitude sectors (one en route and two arrival transition) and one low altitude transition sector. Pilots acted as flight crew of the CDTI-equipped ACFS and of eight single-pilot CDTI-equipped desktop simulators.

Each scenario lasted about 70 minutes. Traffic was a moderate mix of half arrivals and half overflights or departures. A TMA-like scheduler provided a meter fix STA (scheduled time of arrival) for all arrivals, and controllers were asked to meet the STAs as closely as possible. Half of the CDTI-equipped aircraft flown by participants were arrivals and half were overflights. 


\begin{tabular}{|c|c|c|c|c|c|}
\hline \multirow[b]{2}{*}{ Negotiation } & \multicolumn{4}{|c|}{ Condition } & \multirow[b]{2}{*}{ Total } \\
\hline & Baseline & Uplink & $\begin{array}{c}\text { Uplink/ } \\
\text { Downlink }\end{array}$ & $\begin{array}{l}\text { Up/Down } \\
\text { w/ CD\&R }\end{array}$ & \\
\hline Approved on $1^{\text {st }}$ request & 7 & 6 & 5 & 8 & 26 \\
\hline $\begin{array}{l}\text { Rejected on the } 1^{\text {st }} \text { request; uplinked a similar route } \\
\text { before the } 2^{\text {nd }} \text { request }\end{array}$ & 0 & 0 & 2 & 1 & 3 \\
\hline Rejected on 1st request; approved on later request & 3 & 2 & 0 & 1 & 6 \\
\hline Rejected completely & 0 & 1 & 2 & 0 & 3 \\
\hline
\end{tabular}

Table 1. Scripted Pilot-Initiated Negotiation Results

The simulation design planned for 38 pilot-initiated requests. Table 1 summarizes the result of these negotiations between pilots and controllers across the four conditions. In the baseline and the Uplink condition, the negotiation was done by voice, and in the Uplink/Downlink conditions, it was done through CPDLC with voice as a backup channel for conveying pilot and controller intent.

En route controllers approved most of the initial pilot requests and many of the remaining requests were also subsequently approved. The requests that were initially rejected often flew through heavy traffic or into the flight path of another aircraft, so the controllers waited until the paths were clear before accepting the request. An interesting split occurred on the controller actions when the requests were initially rejected and approved at a later time. When the requests were made by voice (i.e. baseline and Uplink), the controller rejected the initial requests and then waited for a second request by the pilot before approving it. In contrast, when the requests were made through CPDLC, the controller viewed the requested route, found some problems with the surrounding traffic, and then immediately uplinked a route that was similar to the downlinked requests but one that also avoided potential conflicts. The data suggests that controllers were more likely to respond in the same modality - i.e. downlinked trajectory with uplinked trajectory and voice request with voice response.

Overall results from this simulation suggest that pilot-controller negotiations of trajectory changes are operationally feasible. Pilots and controllers found the simple procedure adequate, and most requests were approved, if not immediately, then after traffic conditions changed. Controllers only concern was about one potential adverse impact of these silent (off radio) communications: pilots unaware of the controller's CPDLC-mediated interactions with other pilots may overload the controller with requests. Several solutions to this problem were suggested and will be described below.

A satisfactory assessment of the improved controller tools and of air-ground trajectory negotiation feasibility was made under normal operations. This assessment will be described in more detail in the following sections.

\section{Discussion}

\section{A. Air-Ground Trajectory Negotiation}

\section{Voice vs.CPDLC-based trajectory negotiation}

The controllers in our 2003 study had differing views on receiving requests by CPDLC vs. voice. One controller thought that requests through CPDLC should be accompanied by voice, and given a choice, he preferred voice requests. In contrast, another controller strongly favored pilot initiated requests via CPDLC and found the concept to be quite acceptable. On average, controllers had a modest preference for requests made via CPDLC vs. voice.

When comparing trajectory requests made by voice with those made by CPDLC, the requests using CPDLC seem to show certain advantages. They provide clearer intent to the controller than voice requests by specifying the exact route that the pilots want to fly. If the flight deck has $C D \& R$ capability, the requested route can be conflictfree, increasing the probability that it will be accepted. In the 2003 study, the pilots only made simple requests (e.g. "request direct to a waypoint") via CPDLC so that they could be compared to equivalent requests made by voice, but the real benefit of CPDLC-based request may be its ability to design a complex route (e.g. to avoid weather cell, SUAs, etc.), which doesn't have an equivalent counterpart in voice requests.

CPDLC requests potentially have some disadvantages as well. Inherently, visual cues of CPDLC messages on the controller and pilot displays seem to have less salience than voice requests. The visual cues were not always enough to properly alert the controller that a request came in because, when busy, the controller did not notice them. Stronger cues in the data block could be a solution to this problem, but a highly salient cue in the data block may be 
too strong and become a distraction. Another critical factor related to cue salience is the response time. If a fast response time is required for accepting/rejecting the trajectory requests, then CPDLC may be less ideal than voice. In general, however, requests of user-preferred trajectories should not be time critical.

Another interesting difference is the different modality of the requests - i.e. visual vs. auditory. CPDLC does not tie up the radio frequency and therefore low priority requests can be made without blocking more important clearances. In our study, there was also an interesting modality match between pilot requests and the controllers' responses, such that more pilot-initiated requests were modified and accepted via CPDLC than by voice.

\section{Efficacy of the trajectory negotiation process}

In the 2003 study, we minimized the CPDLC-based trajectory negotiation interaction to a) a pilot initiated request via CPDLC, b) a controller response via CPDLC, and c) an accompanying voice response with a rejected request. We also asked the participant controllers and pilots if they could suggest better tools and/or procedures to convey pilot and controller intent during trajectory negotiation. Interestingly, both the pilots and the controllers expressed that a simple interaction was sufficient. Controllers commented that they didn't really need to know why a pilot made a particular request and that often they could guess the intent based on the context. Controllers thought that a simple "unable" or "unable due to traffic" was sufficient communication to convey why a request was rejected. In contrast, pilots wanted to better understand the reason behind the controller action but found the simple controller response acceptable.

This finding was encouraging because efficient trajectory negotiation process is critical to its feasibility. The negotiation process cannot be too long because controllers have other more important tasks that they need to perform. There is also a fixed time frame a CPDLC-based trajectory requests remain valid because a requested route assumes a starting point and the route is invalid if the request is not accepted by that point. In our experiment, the pilot or the controller contacted the other party via voice whenever the time-critical element of the request was not executed by the other party. Whether this is an acceptable solution in the real world is not clear. It is possible to design a system so that eventually a trajectory clearance or request may become invalid (you fly past the turn point). It should be possible for air or ground automation (depending on where the request was initiated) to determine whether the trajectory has the potential to time-out, and if so, when that has occurred or is about to occur. An alert could be provided to the human, or the request could somehow be automatically voided or canceled.

An effective but unforeseen way that the controllers used the ground side tools in our study to accommodate the route requests was to reject the downlinked request but subsequently uplink a route that modified the downlinked request to gain a greater safety margin. This seemed to be an interesting and effective way of accommodating pilotpreferred trajectories without a cumbersome negotiation process.

3. Impact on workload

Overall, the pilot and controller workload associated with trajectory negotiation seemed relatively low. Trajectory negotiations did not seem cumbersome for the controllers. Once they saw the downlinked request, they opened up the route, saw if it was feasible, and either accepted or rejected the clearance. The controllers tried to accommodate the request, workload permitting, and when they were busy, they might have rejected more requests than necessary. Controllers also commented that the ability to data link clearances greatly reduced their overall workload.

Interestingly, from the flight crew's perspective trajectory negotiation is somewhat self-regulating since it is a voluntary activity: only when workload permits will the flight crew initiate a request. In the simulation, the flight crew made route requests and their subjective feedback did not indicate any increase in the workload due to trajectory negotiation. They also commented that CPDLC reduced their workload in the en route environment.

\section{Flight Deck $C D \& R$}

Based on the observations made during the 2003 study, it appears that it is feasible to conduct trajectory negotiations without flight deck CD\&R, although the requests may be accepted more often when the trajectory is conflict-free. The controllers were not always using the ground conflict probe to determine whether a trajectory was clear. They were still using their normal heuristics (is the problem imminent or downstream, etc.). Since controllers seemed to reject some of the conflict-free routes whenever there were alternative routes that were safer, it is not clear how better CD\&R in flight deck would impact controller's decision making in short and long term trajectory planning.

There is a high degree of interdependency between flight deck CD\&R, traffic density, and controller heuristics. Flight deck CD\&R helps the pilot construc conflict-free paths, which might be difficult without CD\&R in high traffic situations. If too many requests with conflicts are made, it could significantly impact controller workload, particularly if pilots send a second, modified request after an initial rejection. On the other hand, conflict-free paths do not guarantee acceptance since a "safe" route by controller standards may require more than missing other 
aircraft by the minimum separation requirement. A flight deck tool that can generate paths that meet the controller's safety heuristics may be needed to substantially increase the fidelity of the request.

5. Potential impact in the field - possible benefits and unintended consequences

Although the trajectory negotiation concept and procedures tested in the 2003 study demonstrated its feasibility and acceptability, potential benefits seemed limited in nominal traffic situations. In current day operations, controllers try to provide services to the pilots by keeping them on an efficient direct routing whenever possible. Potential for maximum benefits seem to reside in off-nominal situations, such as in bad weather. Instead of pilots requesting a heading to avoid weather cells, they could downlink a precise route requesting their preferred path based on current flight deck data. Another off-nominal situation that was mentioned by a pilot was requesting a different altitude based on the ride. These and other off-nominal situations should be explored in future studies.

During debrief discussions in 2003, controllers expressed concerns about a potential negative operational impact on their workload if the system and procedures were not designed properly. In current operations, pilot voice requests have an "automatic" workload filter: when the controller is busy, the communication channel is likely to be saturated and the pilots will not make any requests during that time. However, since they can send data link requests at any time, they are more likely to send requests even during busy times. Since there is no direct person-to-person interaction via data link, pilots may make more requests that might annoy controllers through this medium than by voice. Pilots also expressed an interest in being notified if the controller was busy and could not fulfill their requests. Another potential problem with trajectory requests is that pilots may make route requests that will rarely be honored because they span multiple sectors and require controller-to-controller coordination.

Potential solutions were discussed, such as considering the ability to make requests as a center-wide or sectorspecific service which could be turned on or off based on the traffic complexity and controller workload. Pilots would be alerted on the status of this service upon entering a new sector so that the requests can be made only when the controllers can accommodate them. Another solution is assigning priority to the downlinked requests (weather with high priority, short-cuts with lower priority) to assist in filtering such messages (either by DST or by controller). Low priority requests could even be blocked (with the flight crew notified, of course), or timed out, and drop off the controller's list (again, with appropriate feedback to the flight crew).

\section{B. Air-Ground-Data Link Integration}

1. Controller display

Added display content to support CE 6 operations from the radar controller's workstation inevitably increased clutter. Controllers did not find the level unacceptable, however, and indicated that it was reduced in the 2003 MACS/DSR interface. They also found none of the displayed information to be superfluous.

With one exception (downlink alerts) data block additions appeared to be effective, and controllers did not report any reduction in their ability to monitor traffic. Even the presentation of speed advisories in the fourth line of the data block was well received. In fact, the low altitude (Bowie) arrival controller reported scanning for speed advisories as a means of monitoring STA conformance.

The presentation of message status information (that allows the controller to monitor message status after it is sent) could be improved. We modeled ours after Miami Center's CPDLC I interface, which uses a symbol change in the data block (diamond to up-arrow or lightning bolt) coupled with a status list entry when a message is sent and waiting for the pilot's reply. A status list entry color change (from green to white) is the only cue provided when a message's status requires the controllers' attention (either a negative reply or a "timed out" message). Although this isn't a very salient cue, it's suitable for the type of messages used today in Miami (information and frequency change).

Use of a list format for alerting the controller to time-critical information has potential problems, however, because controllers stop scanning it when they get busy. A more salient cue may help, and should probably be incorporated into the data block. We could, for example, add color coding and/or flashing to the data link symbol to call the controller's attention to a clearance that was no longer valid, or that was rejected by the flight crew. More effective cues to indicate arrival of a downlink request are also needed.

2. Impact on time-based metering

With the better precision and stability provided on meter fix ETA calculations, controllers delivered the simulated aircraft to within seconds of their scheduled meter fix crossing time (STA, or scheduled time of arrival). Schedule-based sequencing was supported by graphically representing ETA-STA difference on a timeline. Controllers quickly learned to scan the timeline for misaligned ETA-STA pairs. A speed advisory appeared in the data block of an arrival aircraft whenever its ETA was more than 15 seconds off its target STA. Controllers could issue speed advisory clearances by voice or data link and found them a very effective metering tool. 
A potentially problematic side effect for time-based metering was observed in this environment. The sensitivity of information presented on the timeline, coupled with the criteria used for displaying speed advisories and the ease of issuing data link clearances may have encouraged controllers to 'overwork' the problem, issuing clearances to correct delays of 5-10 seconds, when 15 seconds tolerance was sufficient.

\section{Impact on trial planning and uplink clearances}

A graphical trial planning tool for lateral route changes allowed the controller to evaluate flight time changes and possible conflicts associated with the new route before assigning it (Figure 2). Trial plans were automatically formatted as uplink clearances that could be sent using a simple command (type "UC" then select the aircraft).

A similar trial plan tool was presented to Fort Worth Center controllers during a field test of the En Route Descent Adviser in 1998 (although that tool was not integrated with CPDLC). Based on its performance, trial planning was rejected as too slow a process for operational use during typical traffic conditions. ${ }^{17}$ Increasing the responsiveness of trial planning was therefore a high priority in software development for the DAG-TM simulations, and controller ratings of the usability and usefulness of the trial planning tools in recent studies reflect progressive, substantial improvement from the 2002 interface. Fast response time and ease of use of trial planning tool, coupled with ease of sending them as clearances via data link, allowed near $100 \%$ of route modifications to be done through data link. In our most recent study, controllers rated the trial planning tool at 5.0 for usability and 5.0 for usefulness on a 5 -point scale.

4. Air-ground communication

Except for check-ins and altitude clearances (CPDLC-based altitude clearances were not available for 2003 study but have been implemented since then), the radios were extremely quiet during the simulation, because controllers effectively shifted most clearances to CPDLC. Unlike 2002 and earlier studies, controllers found CPDLC-based clearances easy to use and preferred to use CPDLC as their default mode of air-ground communication.

While reducing radio congestion is a goal of CPDLC, loss of this "party-line" information (information provided to pilots by overheard communications on a shared radio frequency) also reduced the pilot's awareness of controller workload. No other loss of pilot situation awareness due to this party-line effect was observed, and pilots commented that the CDTI provided far better situational awareness than party-line.

5. Transfer of Communications (TOC)

The use of CPDLC to support transfer of communications deserves discussion not because it's a new idea - it has been used at Miami Center for several years as part of CPDLC I - but because it is so effective. Controllers participating in our 2003 and 2004 simulations were very enthusiastic about it, and our experience supports the assertions based on fast-time simulations that controller workload is significantly reduced, and sector capacity potentially increased by implementation of this function alone. ${ }^{18}$

6. mixed equipage

The impact of mixed equipage on CE 6 was not studied in either of the simulations discussed here. The issue is complex, and definitely needs to be explored. Questions related to this include:

- What variations in CPDLC and ADS-B equipment may be found in an operational environment?

- Can the controller distinguish appropriately between aircraft with differing uplink capabilities?

- Can the controller distinguish appropriately between aircraft with differing information quality?

The mix is not only in percentage, but in type or level of equipage. Aircraft may be equipped with ADS-B but not CPDLC, or support CPDLC for TOC only, or TOC and free text messaging, etc. The equipage options become complex, and can affect controller assessment of information quality and clearance options. Ground automation must be designed to support the controller in making appropriate decisions for each aircraft. It should provide relevant context-specific distinctions based on varying information quality (e.g., conflict prediction quality clearly distinguishable for aircraft with and without ADS-B or intent data). It should also restrict clearance and trial planning options based on the aircraft's message handling capability. Even with well designed automation support, however, the potential complexity of the mixed operational environment could seriously undermine the both the feasibility and benefits of this concept.

\section{Conclusions}

Results from two air-ground simulations suggest that the Distributed Air-Ground Traffic Management concept for integration of air and ground DSTs with data link is feasible and could provide considerable operational benefits. Integration of speed advisory and trial planning DSTs with uplink message formatting and an efficient interface for constructing and sending these clearances appeared to greatly enhance the usability and effectiveness of both the DSTs and of CPDLC. Trajectory negotiation was supported and metering and route clearances were facilitated, with 
the consequence that most aircraft were flying FMS trajectories known to the ground automation. This ability to keep aircraft on 4-D trajectories improves the accuracy of ETAs and conflict predictions used to develop those trajectories, so that the stability and efficiency of the system is improved. This integrated operational environment thus shows promise of providing more benefit than simply a sum of the contributions of each individual component.

Further research is recommended to explore: (1) the impact of mixed equipage on this concept, and (2) changes in controller techniques and methods as their familiarity with data link operations increases. Research on the first topic should determine how the increase in controller task complexity caused by mixed equipage affects concept feasibility and benefits. The second topic should explore how to make best use of these procedural shifts, and further the development of concepts, tools and procedures for trajectory-based operations.

The air-ground negotiation of flight crew trajectory requests appears to be feasible; concerns raised during the 2003 simulation seem straightforward to address. Follow-up studies based on suggestions described in this paper include development and assessment of (1) improved cueing for downlink requests and time-critical or expired messages, and (2) procedures and automation to manage requests based on message urgency and controller workload. Further research is also needed identify benefit scenarios for trajectory negotiation. Weather, complex route optimization requests and efficient user preferred SUA or traffic avoidance are possibilities.

\section{Acknowledgments}

The research described in this paper was supported by the hard work of many individuals, including the flight deck display research group, the MACS development team, the ACFS staff and Ames' Human Factors Division's support group. Contractors Paul Mafera, Jean-Francois D'Arcy, Nicole Racine, Michael Clamann and Greg Niehus contributed substantially to the successful completion of these simulations and their associated reports. We greatly appreciate the support of the Air Line Pilots Association, the National Air Traffic Controllers Association, and the FAA's Air Traffic Services office. This research was sponsored by NASA's AATT project office.

\section{References}

${ }^{1}$ Green S. M., Billimoria, K.D., and Ballin, M.G., "Distributed Air/Ground Traffic Management for En Route Flight Operations," ATC Quarterly, Vol. 9(4), Arlington, VA., 2001, pp 259-285.

${ }^{2}$ AATT Project Office, NASA Ames Research Center, "AATT Concept Definition for Distributed Air/Ground Traffic Management (DAG-TM),” Version 1.0, 1999, Moffett Field CA. URL: http://www.asc.nasa.gov/aatt/.

${ }^{3}$ Couluris, G.J., "Detailed Description for CE 6: En Route Trajectory Negotiation,” NASA Contractor Report NAS2-98005 RTO-41, 1999.

${ }^{4}$ Johnson, W., Battiste, V., and Holland-Bochow, S., "A Cockpit Display Designed to Enable Limited Flight Deck Separation Responsibility," World Aviation Conference, Anaheim, CA, 1999.

${ }^{5}$ Johnson, W., Battiste, V., Delzell, S., Holland, S., Belcher, S., and Jordan, K., "Development and Demonstration of a Prototype Free Flight Cockpit Display of Traffic Information," SAE/AIAA World Aviation Congress Proceedings, 1997.

${ }^{6}$ Prevôt, T., Shelden S., Palmer E., Johnson W., Battiste V., Smith N., Callantine T., Lee P. and Mercer J. "Distributed Air/Ground Traffic Management Simulation: Results, Progress and Plans" AIAA-2003-5602, Reston, VA., 2003.

${ }^{7}$ Prevôt, T. "Exploring the many perspectives of distributed air traffic management: The Multi Aircraft Control System MACS," International Conference on Human-Computer Interaction in Aeronautics, edited by S. Chatty, J. Hansman, and G. Boy, Menlo Park, CA, 2002, pp. 149-154. http://humanfactors.arc.nasa.gov/ihi/research_groups/air-ground-integration

${ }^{8}$ Lee, P., Mercer, J., Prevot, T., Smith, N., Battiste, V., Johnson, W., Mogford, R., and Palmer, E., "Free Maneuvering, Trajectory Negotiation, and Self-Spacing Concepts in Distributed Air-Ground Traffic Management," $5^{\text {th }}$ FAA/Eurocontrol Air Traffic Management R\&D Seminar, Budapest, Hungary, 2003.

${ }^{9}$ Prevôt, T., Lee, P., Callantine, T., Smith, N., and Palmer, E.A., "Trajectory-Oriented Time-Based Arrival Operations: Results and Recommendations," $5^{\text {th }}$ FAA/Eurocontrol Air Traffic Management R\&D Seminar, Budapest, Hungary, 2003.

${ }^{10}$ Lee, P. U., D'Arcy, J. F., Mafera, P., Smith, N., Battiste, V., Johnson, W., Mercer, J., Palmer, E. A., Prevot, T., “Trajectory Negotiation via Data Link: Evaluation of Human-in-the-loop Simulation." International Conference on Human-Computer Interaction in Aeronautics, Toulouse, France, 2004.

${ }^{11}$ Erzberger, H., Davis, T.J., and Green, S.M., "Design of Center-TRACON Automation System," AGARD Meeting on Machine Intelligence in Air Traffic Management, Berlin, Germany, May 1993. http://ctas.arc.nasa.gov

${ }^{12}$ Lockheed Martin, "Display System Replacement/DSR System Support Complex (DSR/DSSC), Air Traffic Controller User Manual," Document TI6160.50, Washington, DC, November 2002.

${ }^{13}$ McNally, B. D., Engelland, S., Bach, R., Chan, W., Brasil, C., Gong, C., Frey, J., Vincent, D., “Operational Evaluation of the Direct-To Controller Tool,: $4^{\text {th }}$ FAA/Eurocontrol Air Traffic Management R\&D Seminar, Santa Fe, NM, December, 2001.

${ }^{14}$ Brasil, C., McNally, D., 2003, "R-side training packet: Direct-to controller tool, DSR R-Side," Direct-To/ URET Integrated Simulation, FAA WJHTC, Atlantic City, NJ, April 22-24 2003. 
${ }^{15}$ FAA, "En route ATCS CPDLC 1 Training," Version 1.02, Atlantic City, NJ, December 2000.

${ }^{16}$ Kopardekar, P., Battiste, V., Johnson, W., Mogford, R., Palmer, E., Smith, N., D’Arcy, J., Helbing, K., Mafera, P., Lee, P., Mercer, J., Prevot, T., and Shelden, S., "Distributed Air/Ground - Traffic Management: Results of preliminary human-in-theloop simulation." AIAA Digital Avionics Systems Conference, Reston, VA, 2003.

${ }^{17}$ McNally D., Erzberger H., Bach R., and W. Chan, "Controller Tools for Transition Airspace," AIAA GNC Conference, Reston, VA, 1999.

${ }^{18}$ Leiden, K., Kopardekar, P. and Green, S., "Controller Workload Analysis Methodology to Predict Increases in Airspace Capacity," AIAA Aviation Technology, Integration, and Operations Forum, Reston, VA, 2003. 